\title{
Peripheral nerve morphogenesis induced by scaffold micropatterning
}

\author{
Federica Cerri ${ }^{\mathrm{a}, 1}$, Luca Salvatore ${ }^{\mathrm{b}, 1}$, Danish Memon ${ }^{\mathrm{c}}$, Filippo Martinelli Boneschi ${ }^{\mathrm{a}}$, \\ Marta Madaghiele ${ }^{\mathrm{b}}$, Paola Brambilla ${ }^{\mathrm{a}}$, Ubaldo Del Carro ${ }^{\mathrm{a}}$, Carla Taveggia ${ }^{\mathrm{a}}$, Nilo Riva ${ }^{\mathrm{a}}$, \\ Amelia Trimarco ${ }^{a}$, Ignazio D. Lopez ${ }^{a}$, Giancarlo Comi ${ }^{a}$, Stefano Pluchino ${ }^{c}$, \\ Gianvito Martino ${ }^{\mathrm{a}}$, Alessandro Sannino ${ }^{\mathrm{b}, *}$, Angelo Quattrini ${ }^{\mathrm{a}}$ \\ ${ }^{a}$ Division of Neuroscience and INSPE, San Raffaele Scientific Institute, via Olgettina 60, 20132 Milan, Italy \\ ${ }^{\mathrm{b}}$ Department of Innovation Engineering, University of Lecce, Via per Monteroni, 73100 Lecce, Italy \\ ${ }^{\mathrm{c}}$ Department of Clinical Neurosciences, Centre for Brain Repair, University of Cambridge, Robinson Way CB2 OPY, UK
}

\section{A R T I C L E I N F O}

\section{Article history:}

Received 6 December 2013

Accepted 26 January 2014

Available online 20 February 2014

\section{Keywords:}

Biomaterials

Peripheral nervous system

Nerve regeneration

Medical device

\begin{abstract}
A B S T R A C T
Several bioengineering approaches have been proposed for peripheral nervous system repair, with limited results and still open questions about the underlying molecular mechanisms. We assessed the biological processes that occur after the implantation of collagen scaffold with a peculiar porous microstructure of the wall in a rat sciatic nerve transection model compared to commercial collagen conduits and nerve crush injury using functional, histological and genome wide analyses. We demonstrated that within 60 days, our conduit had been completely substituted by a normal nerve. Gene expression analysis documented a precise sequential regulation of known genes involved in angiogenesis, Schwann cells/axons interactions and myelination, together with a selective modulation of key biological pathways for nerve morphogenesis induced by porous matrices. These data suggest that the scaffold's microstructure profoundly influences cell behaviors and creates an instructive micro-environment to enhance nerve morphogenesis that can be exploited to improve recovery and understand the molecular differences between repair and regeneration.
\end{abstract}

(c) 2014 Elsevier Ltd. All rights reserved.

\section{Introduction}

Unlike the central nervous system (CNS), peripheral nervous system (PNS) has an innate capacity to regenerate itself in order to fill injuries below a critical size and only if Schwann cell basal lamina integrity is maintained. None-the-less, the regenerated fibers have thinner myelin sheaths than those of normal fibers [1], and never show complete functional recovery. Over the last decades much effort has been made to provide biomaterials that could influence cell behavior in order to efficiently repair injured gaps in peripheral nerves, and to increase our understanding in the molecular differences between regeneration in PNS and CNS, and between development and regeneration. Although biomaterials can direct cells growth and influence specific patterns of gene expression (GE) in cell cultures, tissue regeneration in vivo is a complex event, involving intrinsic and extrinsic factors that are critical for proper tissue development and function [2].

\footnotetext{
* Corresponding author. Tel.: +39 0832 297259; fax: +39 0832297240.

E-mail address: alessandro.sannino@unisalento.it (A. Sannino).

1 Federica Cerri and Luca Salvatore contributed equally to this work.
}

Several studies have already described tissue engineering paradigms that may induce nerve regeneration using organic [3] or synthetic materials [4]. The use of a tubular construct (conduit) reconnecting proximal and distal stumps of a transected nerve is capable of inducing regeneration in vivo, and has been the subject of a large number of investigations that gave different degrees of success [5]. The conduit should protect the site of injury from the infiltration of surrounding cells [6,7], while at the same time should retain a certain degree of porosity allowing diffusion of soluble factors through the tube wall, as well as affecting the migration and organization of myofibroblasts, which are responsible for the undesired synthesis of scar tissue [6]. Thus, the capacity to limit and/ or prevent the formation of the contractile capsule of myofibroblasts, as well as a certain grade of permeability are two key factors which must be carefully considered when working at the improvement of the tube wall properties. Moreover, the orientation of the pores has also been shown to play a critical role in its performance [8-10], together with the biodegradation rate of the conduit [11]. Several studies have focused on the fabrication techniques that create a gradient in pore size along the tube wall [12], combining multiple manufacturing methods in order to increase the level of manipulation in scaffold micro-structures [13]. Even 
though an adequate porosity is considered a critical point for the enhanced nerve regeneration [4], conclusive results about the ability of porous conduit to improve regeneration in vivo are still lacking $[13,14]$.

Although many studies have investigated the effectiveness of nerve conduits in nerve repair, few data have been collected on the molecular mechanisms underlying nerve regeneration inside the conduits. Moreover, whether biomaterials can recreate the complexity of molecular processes that take place during morphogenesis is still an unsolved question as well as one of the major goals of tissue engineering [2].

We have developed a micro-patterned collagen scaffold (MPCS) obtained by means of low cost spinning technique [15], based on thermodynamic and sedimentation phenomena to control pore size gradient and orientation, without the use of any complex mold. The main purpose of our study was to assess the extent of nerve regeneration induced by novel collagen scaffold and to dissect the biological events that occur inside the conduit in rat sciatic nerve repair in vivo. In order to evaluate if and how porous micropatterning might influence nerve regeneration, we performed comparative studies using morphological, functional and gene expression analyses.

\section{Materials and methods}

Experiments with animals followed protocols approved by the Institutional Animal Care and Use Committees.

\subsection{Preparation of scaffolds and surgical procedures}

As reported elsewhere [15], micro-patterned collagen scaffolds (MPCS) were prepared starting from a collagen-based slurry, spun in a proper apparatus, frozen in liquid nitrogen and transferred to a freeze-dryer for lyophilization. Two aqueous suspensions differing for the collagen concentration (either 3\% or 5\% w/v Type-I collagen from bovine dermis, Symatese Biomateriaux) were used, in order to obtain scaffolds with different pore size and pore volume fraction. The two types of scaffolds were referred to as MPCS\#1 ( $3 \% \mathrm{w} / \mathrm{v}$ collagen, high porosity scaffold) and MPCS\#2 (5\% w/v collagen, low porosity scaffold). After freeze-drying, all the scaffolds were cross-linked via a standard dehydrothermal (DHT) process $\left(121{ }^{\circ} \mathrm{C}\right.$, $30 \mathrm{mmHg}, 72 \mathrm{~h}$ ), to slightly strengthen the collagen network by introducing covalent cross-links among the polypeptide chains. Pore diameters were quantified by scanning electron microscopy images of scaffolds.

The biological properties of the two kinds of scaffolds were preliminarily screened in a rat model of sciatic nerve transection. The two types of MPCS $(3+3$ animals for MPCS\#1 and \#2) were implanted in two-month-old female SpragueDawley rats after experimental transection of the sciatic nerve. To implant the scaffolds, rats were anesthetized with chloral hydrate $0.5 \mathrm{~g} / \mathrm{kg}$, the sciatic nerve was exposed and a segment was then cut, immediately after the sciatic notch and before distal sciatic nerve branches to leave a gap of about $10-\mathrm{mm}$ after retraction of the ends. The $15-\mathrm{mm}$ conduit was interposed between the proximal and distal stumps and fixed with a single 11-0 nylon suture. Considering the superior cell infiltration into MPCS\#1 (high porosity) over MPCS\#2 (low porosity), only the former scaffolds were studied further in comparison to commercial collagen conduits-NeuraGen ${ }^{\circledR}$ (CCNG), without any pore size gradient in the wall. Animals were implanted unilaterally with MPCS\#1 $(n=30)$ and with CCNG $(n=24)$.

For crush injury, adult rats were anesthetized with chloral hydrate $0.5 \mathrm{~g} / \mathrm{kg}$, and crush injury was performed as described [16]. After skin incision, the sciatic nerve was exposed and crushed at distal to the sciatic notch for $20 \mathrm{~s}$ with fine forceps previously cooled in dry ice. To identify the site of injury, forceps were previously dropped into vital carbon. The nerve was replaced under the muscle and the incision sutured $(n=9)$

\subsection{Neurophysiological and morphological analyses}

To evaluate the degree of regeneration and remyelination after peripheral nerve transection, we performed: (i) longitudinal sciatic nerve conduction studies on rats following implantation of both conduits at 90 and 120 days; (ii) serial morphological studies at different levels on MPCS- and CCNG-implanted rats at 8, 15, 40, 60, 90, 120 days after the implant. The timeline has been selected on the basis of the biological events known to occur during nerve repair process [17]. To study nerve conduction velocity, rats were anesthetized with chloral hydrate $0.5 \mathrm{~g} / \mathrm{kg}$ and placed under a heating lamp to avoid hypothermia. Sciatic nerve motor conduction velocity was obtained with steel monopolar needle electrodes: a pair of stimulating electrodes was inserted subcutaneously near the nerve at the ankle; and a second pair of electrodes was placed at the sciatic notch, to obtain two distinct sites of stimulation, proximal and distal, along the nerve. Compound motor action potential was recorded with an active electrode inserted in the muscles in the middle of the paw and a reference needle in the skin between the first and second digits. Nerve conduction velocity was evaluated at 90 and 120 days after the implant: 3 animals implanted with MPCS and 3 animals with CCNG were examined at the selected time points and compared to 2 uninjured rats (wt), bilaterally recorded.

For the morphological analysis, animals ( $n=2$ wt rats as control, sciatic nerves were retrieved bilaterally; 5 MPCS- and 4 CCNG-implanted rats per time point) were sacrificed by $\mathrm{CO} 2$ inhalation and tissues were removed and fixed with $2 \%$ glutaraldehyde in $0.12 \mathrm{~m}$ phosphate buffer, postfixed with $1 \%$ osmium tetroxide and embedded in Epon (Fluka). Semithin (0.5-1 $\mu \mathrm{m}$ thick) and ultra-thin (100-120 nm thick) sections were obtained and examined by light (Olympus BX51) and electron microscopy (Leo 912 Omega). Sciatic nerves were divided at different levels to ensure the study of the inside of the tube, even after it had been reabsorbed $(+2,+4$ and $+8 \mathrm{~mm}$ from the proximal stump). To establish the process of reinnervation at the distal part, together with sciatic nerves, we also investigated the tibial plantar nerves at the paw. Digitalized images of fiber cross-sections were obtained from corresponding levels of the sciatic nerve with a digital camera (Leica DFC300F) using a $100 \times$ objective. Morphometry on semithin sections was analyzed with the Leica QWin software (Leica Mycrosystems, Milano, Italy) [18]. The ratio between the mean diameter of an axon and the mean diameter of the fiber including myelin ( $g$-ratio) was determined on at least 300 randomly chosen fibers per group ( 3 animals each MPCS, CCNG and controls) from electron microscopy images [19] taken at different levels inside the conduits and at the peroneal branch of sciatic nerve. Morphometric data differences between control nerves, MPCS and CCC-NG were analyzed by OneWay ANOVA followed by Bonferroni post-hoc test. Statistical significance was considered at $p<0.05$. All statistical tests were performed using SPSS software (Technologies, Inc., Chicago, IL, USA).

\subsection{RNA extraction and gene expression analysis}

To investigate the molecular changes in each of the experimental groups, we performed whole genome expression profiling study with Illumina ${ }^{\circledR}$ RatRef-12 Expression Beadchips on MPCS and CCNG-implanted rats at 8, 25 and 40 days post-surgery, compared to sciatic nerve after crush injury and from healthy control rats. Together with MPCS- and CCNG-implanted rats, for gene expression analysis we chose the crush injuries as the control of efficient and accurate regeneration because the basal lamina surrounding the axon/Schwann cells nerve unit is preserved and the integrity of the original paths inside the endoneurium was maintained.

Total RNA was isolated from the sciatic nerves of additional animals, at 8, 25 and 40 days (sciatic nerves were retrieved bilaterally from 2 wt rats as control, from 4 MPCS- and 4 CCNG- unilaterally implanted rats and 3 crushed nerves, for each time point) using the RNeasy kit (Qiagen). RNA was quantified using the Nanodrop-2000 spectrophotometer (Celbio) and Agilent 2100 Bioanalyzer was used to assess RNA integrity. For genome expression (GE) profiling study we used Illumina ${ }^{\circledR}$ RatRef-12 Expression Beadchips. Each individual array on the chip targets more than 21,000 transcripts selected primarily from the NCBI RefSeq database (Release 16) and in minor part from the UniGene database. Total RNA (500 ng) was reverse transcribed into cRNA and biotin-UTP labeled using the Illumina TotalPrep RNA Amplification Kit (Ambion). We quantified the cRNA with three replicate measurements using Nanodrop-2000 spectrophotometer. cRNA (750 ng) in $11 \mu \mathrm{l}(150 \mathrm{ng} / \mu \mathrm{l})$ were hybridized to the BeadChip Array at $58{ }^{\circ} \mathrm{C}$ overnight. The fluorescent signal was developed through a streptavidin-Cy3 staining step. BeadChips were imaged using the Illumina ${ }^{\circledR}$ BeadArray Reader, a two-channel $0.8 \mu \mathrm{m}$ resolution confocal laser scanner. The software Illumina ${ }^{\circledR}$ GenomeStudio (2010.1) was used to assess fluorescent hybridization signals and the system quality controls, such as biological specimen, hybridization, signal generation and negative controls. Each sample was tested in technical and biological replicates, and the mean correlation coefficient value of technical replicates was 0.991 (SD: 0.004) and of the biological ones was 0.953 (SD: 0.03). Sample clustering analysis based on the absolute correlation metric parameter was performed. The graphical representation of the dendrogram further supported the technical validity of the data.

Initial pre-processing and variance stabilizing normalization of Bead summary data was done using Lumi package [20] in R. The normalized expression values of samples were used to identify differentially expressed genes at different time points of the experiments.

Genes were classified as differentially expressed based on a fold change with a cutoff of 2.0 and adjusted $p$ value less than 0.01 to maintain a low false discovery rate. Log fold change and moderated $t$-statistics were determined based on multiple linear models built using Limma package [21] in Bioconductor. The gene ontology/ biological pathway enrichment analysis was done using DAVID [22,23] and Gene Ontology database [24]. Functional annotation clustering was performed for enriched biological processes at gene ontology level 5 and representative biological process from each cluster was selected. The gene co-expression networks were built from Pearson correlation coefficients between genes with a hard threshold of 0.95 and visualized in Cytoscape tool [25]. The over-represented transcription factor binding sites in co-regulated genes, were obtained from cREMaG database [26].

\subsection{Quantitative real-time PCR analysis}

Differential expression of MBP, P0 and GFAP at 25 days post-surgery was validated by quantitative Real Time polymerase chain reaction (qRT-PCR). In C1000 
Thermal Cycler with CFX96 Real-Time System (Biorad), using SYBR Green I as the detection format, steady state levels of mRNA encoding MBP, P0 and GFAP were selected for validation.

We synthesized cDNA using High Capacity cDNA Reverse Transcription Kits (Applied Biosystems) according to manufacturer's instructions and amplified the following primers pairs: 5'-GTAACATGCAAGAAACAGAAGAGTG-3' (forward) and 5'TAGCTTAACGTTGAGTAGATCCTGG-3' (reverse) for GFAP (final PCR product $329 \mathrm{bp}$ ); 5'-CACAACCTAGACTACAGTGACAACG (forward) and 5'-TTCGAGGAGTCCTTAGAAGACTTG-3'(reverse) for P0 (final PCR product 290 bp); 5'-3' ACACACAAGAACTACCCACTACGG (forward) and 5'- GTACGAGGTGTCACAATGTTCTTG-3' (reverse) for MBP (final PCR product $105 \mathrm{bp}$ ). MBP primers were designed in the genomic regions common to all 5 isoforms of the gene in order to evaluate total MBP expression. Expression of all genes was compared to GAPDH expression, used as housekeeping gene in our analysis. Two different primers specific for GAPDH were designed to obtain the same annealing temperatures and final lengths for PCR products. Primers pair 5'- ACCTCAACTACATGGTCTACATGTTC-3' (forward) and $5^{\prime}$ ATCTTGAGGGAGTTGTCATATTTCTC $-3^{\prime}$ (reverse) with annealing temperature of $60{ }^{\circ} \mathrm{C}$ and a final PCR product 322 bp long was used as housekeeping gene in qRTPCR experiments for GFAP and MPO. Primers pair 5'-CTGAGTATGTCGTGGAGTCTACTGG $-3^{\prime}$ (forward) and $5^{\prime}$ - GTCATATTTCTCGTGGTTCACACC $-3^{\prime}$ (reverse) with annealing temperature of $61{ }^{\circ} \mathrm{C}$ and a final product 149 bp long was used as housekeeping gene in qRT-PCR experiments for MBP.

For PCR reactions Sso fast EvaGreen Supermix (Biorad) was used according to manufacturer's instructions. On the basis of standard curves analyses for each reaction (made in triplicate), we used a cDNA concentration corresponding to starting RNA concentration of $2 \mathrm{ng}$ for MBP and P0 and $20 \mathrm{ng}$ for GFAP and fina primer concentration was $5 \mu \mathrm{M}$. PCR cycling conditions for GFAP and P0 were: $5 \mathrm{~min}$ at $95{ }^{\circ} \mathrm{C}, 30 \mathrm{~s}$ at $95{ }^{\circ} \mathrm{C}, 30 \mathrm{~s}$ at $60{ }^{\circ} \mathrm{C}$ and $1 \mathrm{~min}$ and $30 \mathrm{~s}$ at $72{ }^{\circ} \mathrm{C}$ (41 cycles). PCR cycling conditions for P0 were: $5 \mathrm{~min}$ at $95^{\circ} \mathrm{C}, 30 \mathrm{~s}$ at $95^{\circ} \mathrm{C}, 30 \mathrm{~s}$ at $61^{\circ} \mathrm{C}$ and $45 \mathrm{~s}$ at $72{ }^{\circ} \mathrm{C}$ (41 cycles).

\section{Results}

\subsection{Optimal micropatterning of MPCS}

As detailed elsewhere [15], the micro-patterned collagen scaffolds used in this study were fabricated by means of a spin-casting or centrifugal casting technique, followed by lyophilization. The technique allows for the production of highly porous tubular constructs, characterized by a radially oriented pore structure in the tube wall (defining radial tracks that could work as directional guidance cues for cells) and a gradient in pore size and relative density along the tube radius. To select the ideal MPCS for this study, we tested in vivo two types of MPCS with radially oriented pore distribution, respectively. MPCS \#1, produced starting from a $3 \% \mathrm{w} / \mathrm{v}$ collagen slurry and MPCS\#2, obtained from $5 \% \mathrm{w} / \mathrm{v}$ collagen slurry. Although a detailed and quantitative analysis of the pore size along the tube radius was not performed, SEM micrographs on different samples (Fig. 1A-C) clearly showed that, while the bulk of the MPCS displays radially aligned pore channels and a mean pore size of approximately $20 \mu \mathrm{m}$, with the region near the inner tube

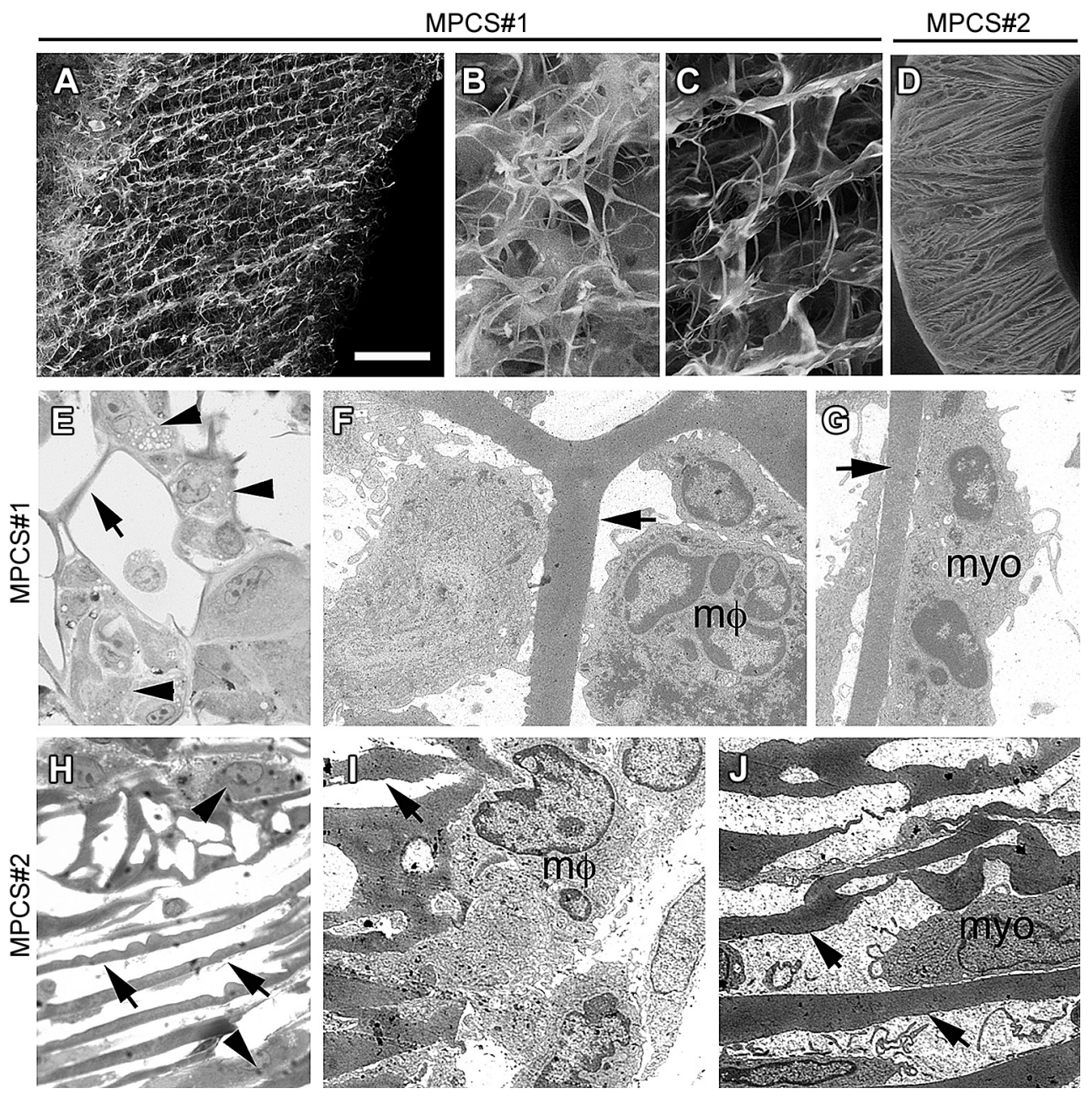

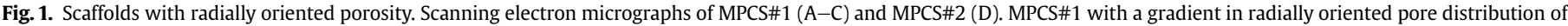

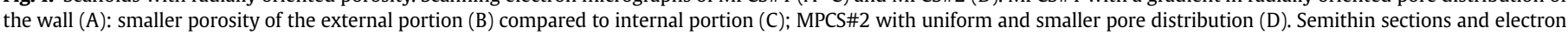

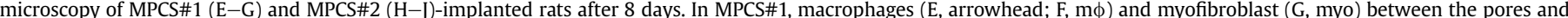

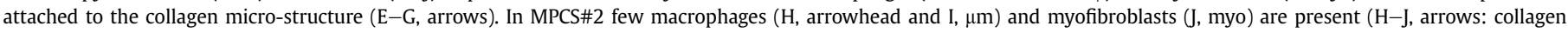
micro-structure). Scale bar: in A and D $100 \mu \mathrm{m} ; 20 \mu \mathrm{m}$ for B, C, E and H; $2 \mu \mathrm{m}$ for F, G, I and J. 
wall characterized by a decreased scaffold density and a less organized structure, the MPCS structure near the outer edge of the tube displays a considerably higher solid volume fraction with a significantly smaller mean pore size $(<5 \mu \mathrm{m})$. The internal region of the tube wall forms a cell-permeable region that may allow cells to migrate into the scaffold from the internal tube lumen, the external region of the tube wall forms a cell-impermeable shell that blocks cell infiltration into the scaffold from the outside, while maintaining protein/oxygen permeability. Morphological studies showed preferential inflammatory cells and myofibroblasts migration from the inner lumen toward the edge of the outer tube in MPCS \#1 (Fig. 1E-G). MPCS \#2 showed smaller mean pore diameter $(5 \mu \mathrm{m})$ (Fig. 1D). The micro-structure of MPCS \#2 did not allow efficient migration of cells from the tube wall (Fig. $1 \mathrm{H}-\mathrm{J}$ ). Thus, for our study, we implanted only MPCS \#1 compared to commercially available collagen conduit Neuragen ${ }^{\circledR}$ (CCNG), the latter not showing any specific micropatterning in the tube wall.

\subsection{Motor functions recovery}

All animals in the 2 groups (MPCS, CCNG) recovered well from the surgery. Few animals from both groups showed a tendency for auto-mutilation of their treated digits, but with no significant difference among the groups. Walking track analysis was performed at 30, 60 and 90 days; at all time points, there was no significant difference between groups. Neurophysiological analysis confirmed that regenerating axons reached the paw in both MPCS- and CCNGimplanted rats as we recorded a compound motor action potential (cMAP) at the paw at 90 days. The mean distal latency in MPCS was lower than CCNG $(1.54 \mathrm{mV} \pm 1.19 ; 2.07 \mathrm{mV} \pm 0.15$ respectively); nerve conduction velocity (NCV) was higher in MPCS $(30.42 \mathrm{~m} /$ $\mathrm{s} \pm 7.06)$ as compared to CCNG ( $18.43 \mathrm{~m} / \mathrm{s} \pm 3.41)$. At 120 days, no significant increase in cMAP amplitudes was found between the two groups; NCV remained higher in MPCS with a mean value of $31 \mathrm{~m} / \mathrm{s} \pm 4$ versus $21 \mathrm{~m} / \mathrm{s} \pm 3$ in CCNG. Although not statistically significant, a trend toward better value for the tested neurophysiological parameters in MPCS-implanted rats was observed.

\subsection{Formation of the clot, angiogenesis and Schwann cell (SC) differentiation}

Within 8 days after surgery, a fibrin clot in MPCS-implanted nerves was present, with the infiltrated blood cells and fibroblasts (Fig. 2A), these producing collagen fibers throughout the MPCS (Fig. 2B and C) in the absence of major scaffold contraction. Early stages of angiogenesis characterized by small capillaries, preferentially localized in the midgraft were documented (Fig. 2D). Ultrastructural studies and immunofluorescence analysis for the tight junction marker VE-cadherin identified tight junctions between endothelial cells (Fig. 2E and F), demonstrating the reestablishment of a physiological nerve-blood barrier. CCNG midgraft contained only few isolated cells (Fig. 2G and H) and vessels without clear tight junctions formation.

To explore the biological functions induced by MPCS on cells at the very beginning of the nerve morphogenesis process, we compared the mRNA expression profile of key molecules to uninjured nerves, CCNG-implanted nerves and crushed nerves (the standard experimental model of nerve regeneration). Microarray analysis showed an up-regulation of transforming growth factor (Tgf)- $\beta 1$ in both MPCS and CCNG groups. The expression of vascular endothelial growth factors (Vegf), a crucial molecule for angiogenesis and nerve regeneration [27], was also up-regulated in both groups (Fig. 2I). However, Vegf mRNA levels decreased progressively in MPCS, reaching nearly the level of intact nerve at 40 days. In crushed nerves, Vegf expression showed no major changes (Fig. 2I).
To monitor the presence of SC in the regenerating areas, we studied the expression of Glial Fibrillary Acidic Protein (Gfap), the marker of denervated SC, at mRNA and protein levels. At 8 days, Gfap mRNA expression inside MPCS was very low (Fig. 2I), consistent with few migrating SCs across the scaffold. At 25 days, Gfap mRNA levels were maximally expressed, reflecting the large amount of SCs inside the MPCS with a subsequent down-regulation at 40 days (Fig. 2I). These data suggest that the MPCS may allow SC migration and proliferation through the conduit as confirmed by increasing levels of mRNA at 8 and 25 days after the implant; the subsequent Gfap down-regulation could represent the SC differentiation phase toward a mature phenotype, in which Gfap expression is physiologically silenced. In crushed nerves, the Gfap expression was already high at 8 days, because of the great number of denervated SCs, peculiar of this early time point, and was returning to normal levels of expression at 40 days (Fig. 2I). In CCNG-implanted rats, Gfap expression remained low at all examined time points. Of note, another key molecule, the EphB which mediates the signaling between fibroblasts and SC (Fig. 2I), directing nerve regeneration through SC sorting [28], was strongly up-regulated at 25 days in the MPCS group, with a subsequent down-regulation to close to normal level at 40 days.

\subsection{Axonal growth and SC/axon interactions}

To characterize axonal growth, we investigated the midgraft 15 days after the implantation. In MPCS, morphological and immunofluorescence analyses showed many regenerating axons inside the conduit interacting with SC (Fig. 3A-C). Of note, we observed the deposition of a basal lamina surrounding the SCs (Fig. 3D). Over time, the SCs sort, enwrap and myelinate axons, depending on the expression of key molecules as integrins, dystroglycan receptors and endoneurial laminins present on basal lamina $[29,30]$. Accordingly, by GE analysis, we found an increased expression of integrin- $\beta 1$, dystroglycan receptors and basal lamina components (laminin- $\beta 1$ and laminin-alpha2) at 25 days in both MPCS and crush groups (Fig. 3E), indicating that SC had re-established a contact with axons and are prone to remyelinate.

\subsection{Initiation and progression of myelination}

To investigate the myelination process, we performed morphological analysis 40 and 60 days after surgery. At day 40, the MPCS collagen wall was not identifiable by both macroscopic and microscopic analyses, matching the rate of nerve formation and collagen re-absorption. While in CCNG-implanted rats, the collagen conduit was still present. As documented by ultrastructural analysis, all fibers with a diameter $>1 \mu \mathrm{m}$ were myelinated with few wraps or with already compact myelin; endoneurium and perineurium were also well organized without inflammatory cells (Fig. 4A-F). Perineurial cells showed typical tight junctions and pinocytic vesicles (Fig. 4F). In CCNG group, the tube wall and the associated inflammatory response were still present; few thin myelinated fibers and many naked axons were evident (Fig. 4G-K) grouped in minifascicles; there were no evidence of structural integrity of endoneurium and perineurium (Fig. 4G-I).

GE analysis showed that integrin- $\beta 4$, which mediates the interactions with extracellular matrix (ECM) [31], was up-regulated at the beginning of myelination in both MPCS and crushed nerves, reaching levels of the intact nerve at 40 days (Fig. 4L). Whereas the expression was always very low in CCNG. Furthermore, desert hedgehog (Dhh), a key player for structural and functional integrity of PNS [32], whose absence causes microfasciculation and abnormal perineurium, increased between 8 and 25 days only in MPCS-implants and crushed group (Fig. 4L). 

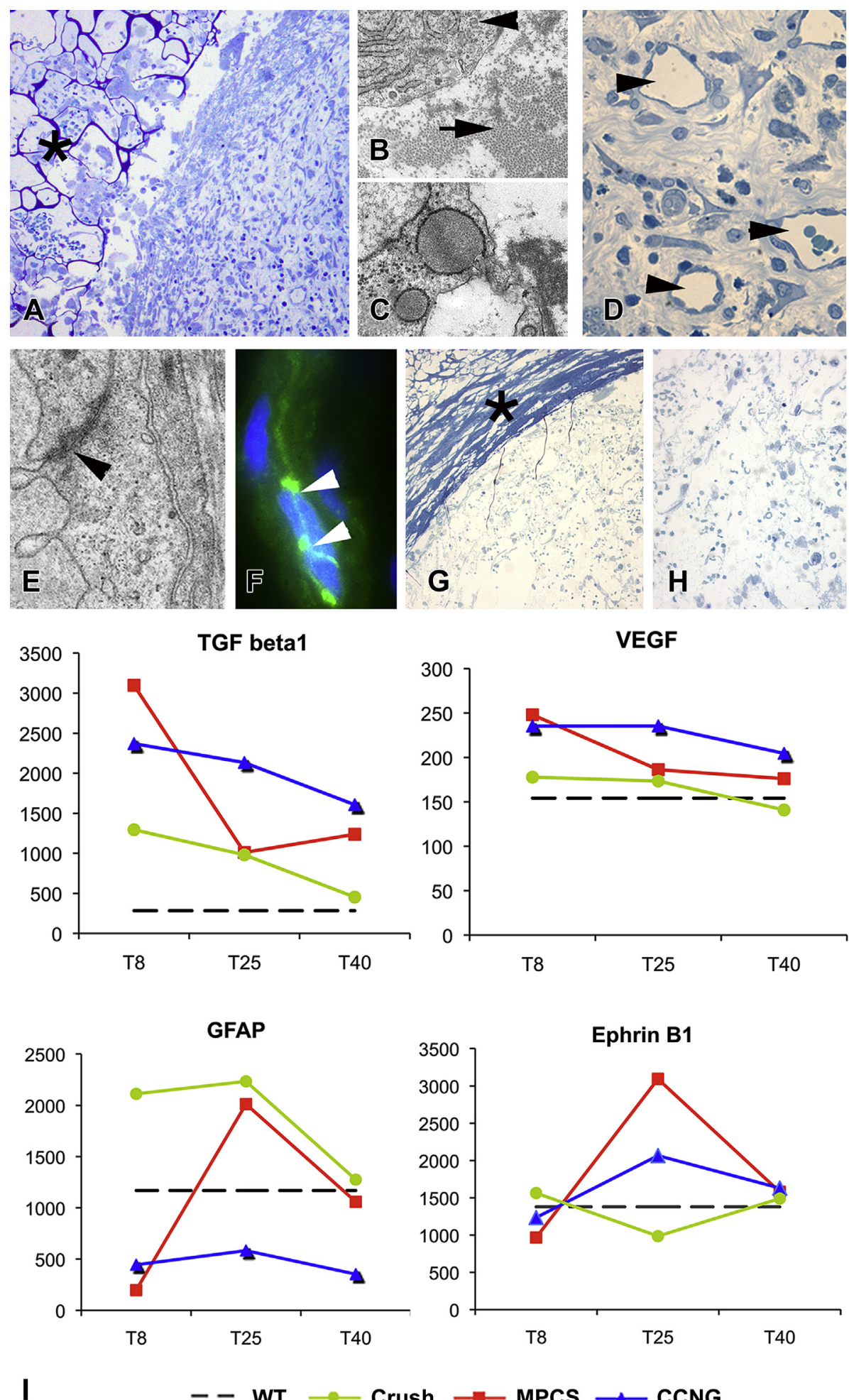

I

- WT $\rightarrow$ Crush

MPCS

CCNG

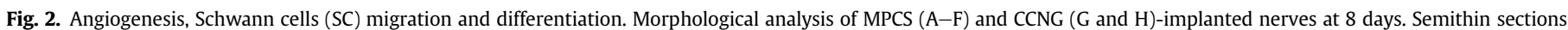

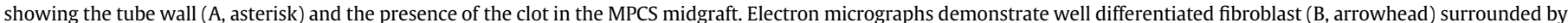

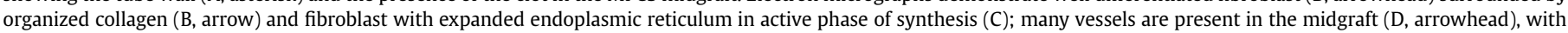

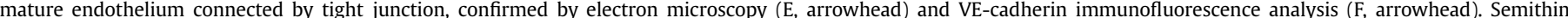

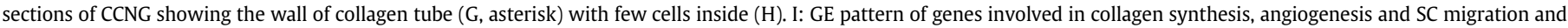
differentiation at the selected time points. Scale bar: $100 \mu \mathrm{m} \mathrm{A}$ and G; $20 \mu \mathrm{m} \mathrm{D}$ and H; $5 \mu \mathrm{m} \mathrm{F;} 1 \mu \mathrm{m} \mathrm{B}, \mathrm{C}$ and E.

Interestingly, the mRNA levels for key regulators of myelination, such as ErbB2/B3 receptors for Neuregulin-1 type-III (NRG1 typeIII) [33,34], continued to increase significantly between 8 and 25 days only in the MPCS-implants (Supplemental Fig. S1).
Of relevance, in MPCS conduits 60 days after implant we observed a normal nerve characterized by normal fiber density, such as intact nerves (Fig. 5A-D and E-I). Only few clusters of thin myelinated nerve fibers, the hallmark of axonal regeneration, were 

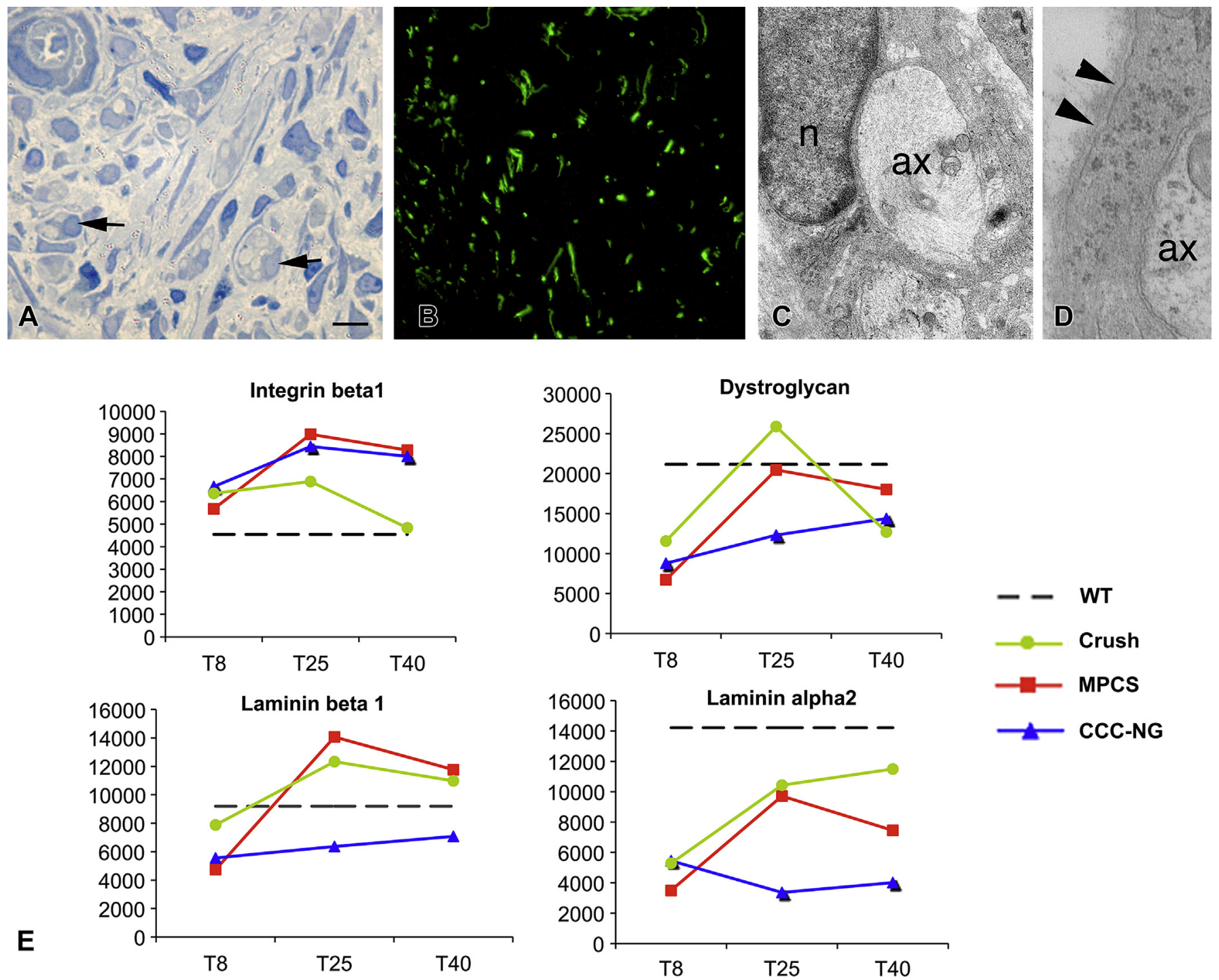

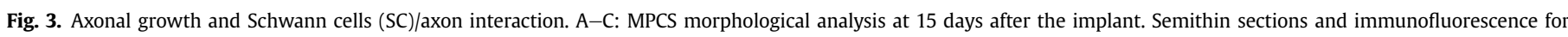

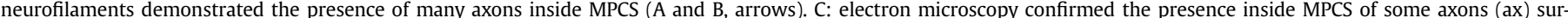

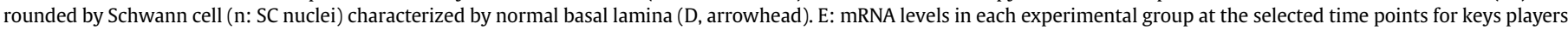
for SC/axon (integrin beta1) and SC/extracellular matrix (dystroglycan, laminin beta1 and laminin-alpha2) interactions. Scale bars: $10 \mu \mathrm{m}$ A; $1 \mu \mathrm{m}$ B and C.

still present in the epineurium (Fig. $5 \mathrm{H}$ ), suggestive of impaired myelination outside the MPCS. Ultrastructurally, the myelin thickness was normal, as well as the morphology of non-myelinated nerve fibers (Fig. 5I). In CCNG-implants, we observed again a delay in myelination, as well as abnormal endoneurium with minifascicles (Fig. 5J-M). Morphological analysis of sciatic nerves outside both scaffolds showed many clusters of fibers with thin myelin compared to wt nerves (Supplemental Fig. S2A-C), suggesting that the regeneration process has reached the distal target and further highlighting the significant regeneration we obtained inside MPCS conduits.

To further confirm these results, we determined the fiber density. Post-hoc comparison showed that fiber density was significantly lower in CCNG compared to both wt $(p=0.001)$ and MPCS $(p<0.0001)$, but did not differ between wt and MPCS (Fig. 5N). We then analyzed myelin sheath thickness by electron microscopy and $g$-ratio measurements. $G$-ratio values were significantly different between MPCS and CCNG at the site of device implant ("MPCS-I" and "CCNG-I" respectively) and between MPCS and CCNGimplanted rat at the level of peroneal branch ("MPCS-O" and
"CCNG-O"). Interestingly, no significant difference was found between MPCS and wt groups at the site of device implant, while the $g$-ratio of peroneal branch of MPCS-implanted rats was higher than control value, demonstrating the presence of thinner myelinated fibers outside the conduit (Fig. 5N). These findings indicate that micro-structure of MPCS scaffold may favor the myelination, while regulating axonal diameter and myelin thickness inside the conduit only.

To determine whether the myelin protein content parallels the myelinating phenotype, we evaluated the expression level of genes involved in the processes of myelination, namely myelin protein zero (Mpz), myelin basic protein (Mbp) and peripheral myelin protein 22 (Pmp22) [35]. Mpz, Mbp and Pmp22 mRNA were upregulated in both crush and MPCS groups with the highest mRNA level in MPCS at 25 days (Fig. 50). Of note, mRNA expression levels of these myelin proteins reached normal values only in MPCS whereas in CCNG-implants they were always low (Fig. 50). To validate the GE data, we performed qPCR for Gfap, Mbp and Mpz on nerve RNA from crush, MPCS and CCNG 25 days after the implant, confirming a similar pattern of expression (Supplemental Fig. S3). 

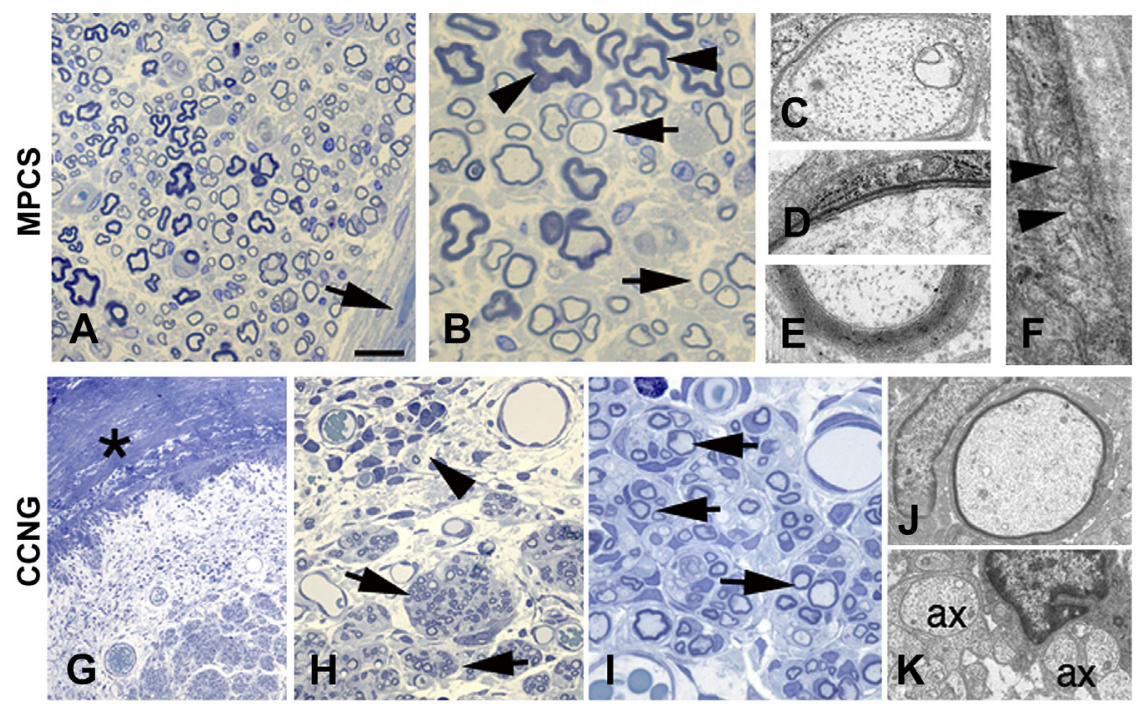

Integrin beta 4
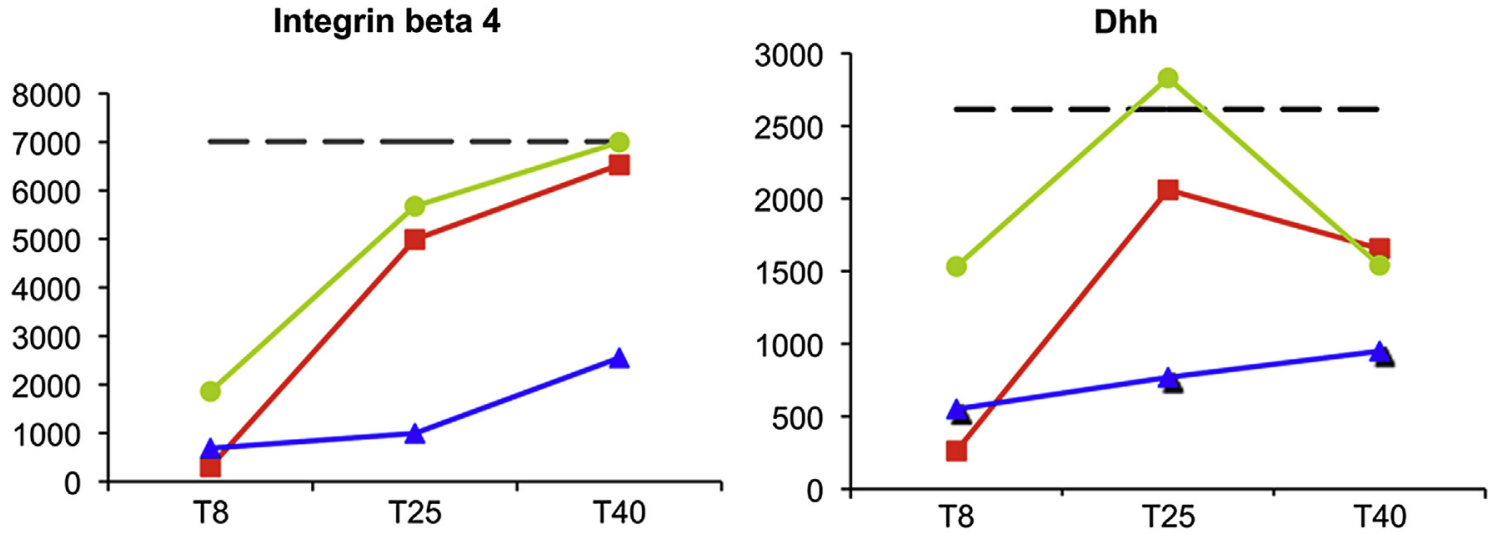

$\mathbf{L}$

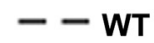

Crush
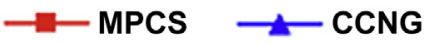

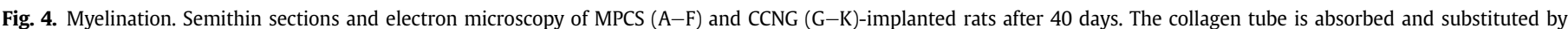

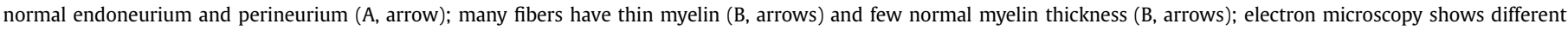

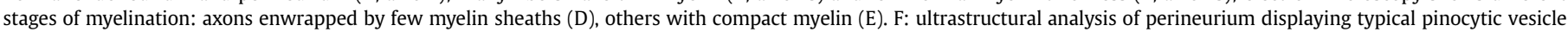

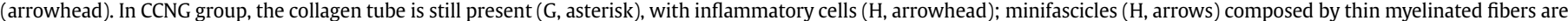

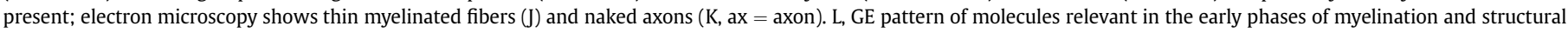
integrity of PNS. Scale bar: $100 \mu \mathrm{m} \mathrm{F} ; 20 \mu \mathrm{m} \mathrm{A}$ and G; $10 \mu \mathrm{m} \mathrm{B}$ and $\mathrm{H} ; 2 \mu \mathrm{m} \mathrm{C}, \mathrm{D}, \mathrm{E}, \mathrm{I}$ and J.

\subsection{The end point of regeneration}

To assess the final step of regeneration process, we also analyzed, together with the sciatic nerve, the plantar branches of the tibial nerve at the paw at 90 and 120 days post-surgery. We observed reinnervation of the distal branches in both MPCS and CCNG group, as documented by the presence of thinner myelinated fibers joined together in clusters (Supplemental Fig. S4A-C). However only in MPCS-implants, the plantar nerves showed normal epineurium and perineurium (Supplemental Fig. S4A), all axons were myelinated and grouped in clusters, indicating active regeneration (Supplemental Fig. S4A) with no signs of axonal degeneration. In CCNG-implants, we found signs of axonal degeneration and abnormal perineurium (Supplemental Fig. S4C and D).

\subsection{Regulation of biological processes at mRNA level}

Given the striking regeneration we observed in the MPCSimplanted rats, to gain more insights into molecular mechanisms underlying nerve regeneration induced by scaffold implant, we assessed GE profiles of MPCS-, CCNG-implanted nerves as compared to crushed and intact WT nerves at the selected time points. First we analyzed the overall behavior at mRNA level. At 8 days from surgery, a larger number of genes underwent significant modulation in MPCS as compared to others (334, 17.4\% of differentially regulated genes) (Supplemental Fig. S5A) with a drop at 25 days as compared to CCNG (634 genes; 41.1\%) (Supplemental Fig. S5B). Nevertheless, at the same time point, the number of genes induced by MPCS was still higher than the crush group, with a decrease at 40 days in both MPCS and crush groups (Supplemental Fig. S5C). This overall analysis suggested that MPCS leads to earlier expression of large number of genes likely correlated to earlier signaling. A total of 228 RNA species at 25 days were found to be exclusively up-regulated in MPCS, compared to CCNG, we found. Interestingly, 8 of them are involved in myelination (Dhh, Lama2, Cldn1, Mbp, Brn-2, meteorin, gliomedin and Lamb2) [3638]. Furthermore, among them, we identified a total of 13 upregulated genes involved in neurogenesis and/or organ morphogenesis (Sox10, Ahd2, BhlhB5, Bmp7, Csda, Col2a1, gpc3, Plp, Rdh10, Schp1, Tncc, Lamb1, Igf2). Compared to crushed nerves, MPCS upregulated a total of 132 RNA species at 25 days, some involved in neurogenesis, organ morphogenesis and/or axiogenesis (Evl, Gfra3, 

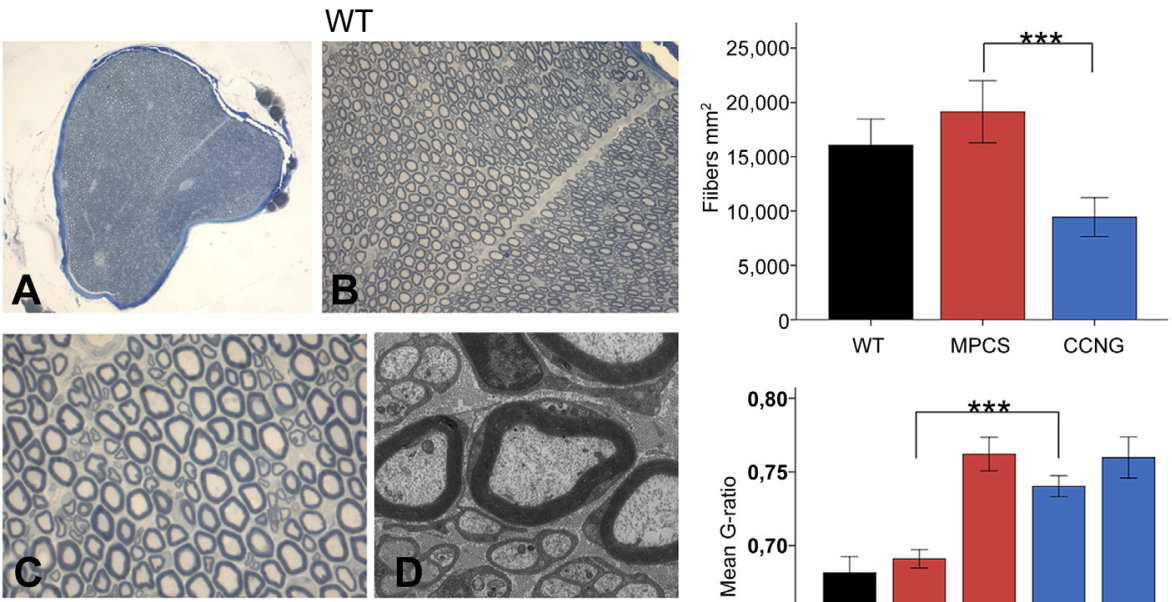

MPCS
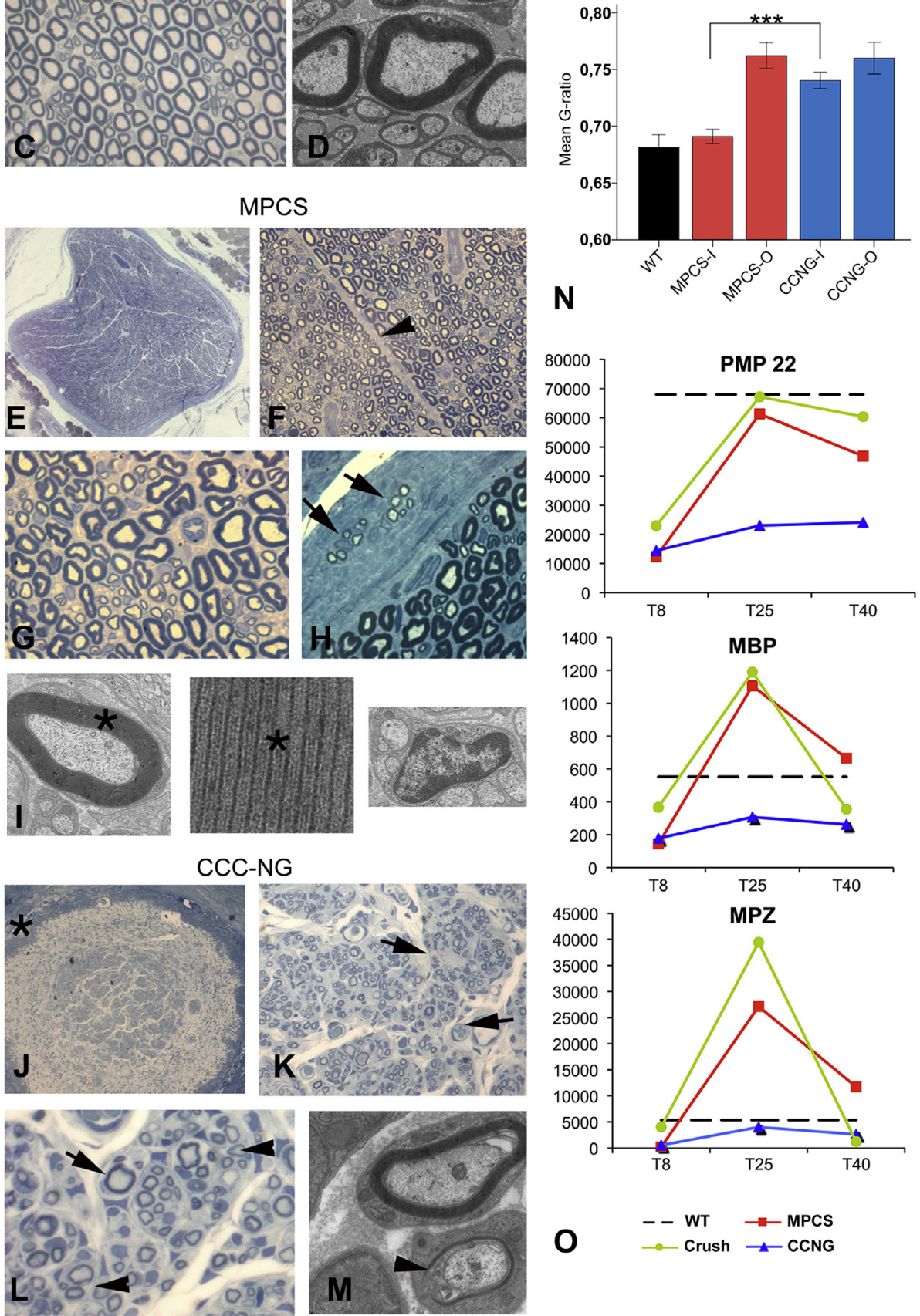

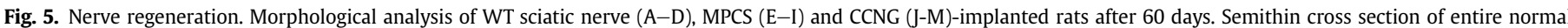

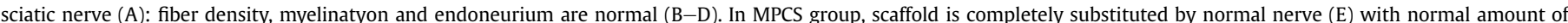

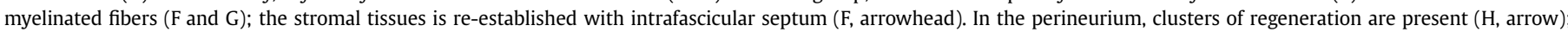

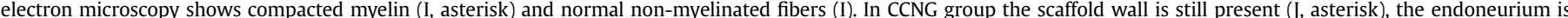

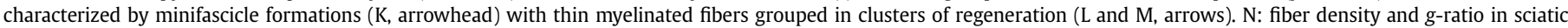

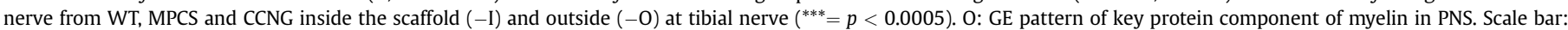
$100 \mu \mathrm{m} \mathrm{A}, \mathrm{E}$ and J; $40 \mu \mathrm{m} \mathrm{B}, \mathrm{F}$ and $\mathrm{K} ; 20 \mu \mathrm{m} \mathrm{C}, \mathrm{G}, \mathrm{H}, \mathrm{L}, \mathrm{N}, \mathrm{O}$ and $\mathrm{P} ; 2 \mu \mathrm{m} \mathrm{D}, \mathrm{I}$ and $\mathrm{M}$. 
Thy1, Bhlhb5, Cspg4, Lerk2, Col8A1, Fhl2). Interestingly, some genes induced by MPCS were a component of Wnt (Gpr177, Tmem88, Dact1) and retinoid acid (Rdh10, Rxrg) pathways, both involved in nervous system development as potent regulators of morphogenesis $[39,40]$.

The co-expression network built from the 48 RNA species upregulated exclusively by MPCS (as compared to either CCNG or crush nerves) had 41 nodes and 80 interactions and was particularly enriched with biological processes related to neurogenesis. Five genes involved in neurogenesis (Ndn, Dbi, Cspg4, Rxrg, Bmp7) and two genes involved in organ morphogenesis (Rdh10, Bhlhb5) were found to be part of the co-expression network (Fig. 6). Analysis of transcription factor binding sites showed high enrichment of binding sites for transcription factors involved in developmental processes. This data suggested that MPCS somehow modulated several molecular pathways related to neurogenesis and organ morphogenesis.

\section{Discussion}

In this study we demonstrated how the peculiar micro-porosity and the selected degradation rate of the collagen scaffold may efficiently influence the biological processes involved in nerve morphogenesis in a rat model of sciatic nerve traumatic injury. We observed an early development of vessels and SC migration, and axonal elongation leading to an efficient process of myelination. With gene expression, we confirmed that MPCS positively modulates known genes involved in PNS regeneration, and above all may selectively induce mRNA species known to be potent regulators of nervous system development.

Over the last decades, several conduits have been developed showing different extent of nerve regeneration. In our experimental setting, both MPCS and CCNG scaffolds allowed nerve regeneration with distal reinnervation, and neurophysiological studies confirmed an early functional recovery with recordable cMAP 90 days after the implant. Although distal reinnervation is somehow reachable with a large variety of experimental conduits, one of the un-answered questions is whether scaffolds used in nerve regeneration can direct cell proliferation and differentiation, ultimately leading to a normal nerve.

During nerve regeneration, multiple genes must be activated in the correct sequence and synchrony in order to guarantee a balanced expression of proteins needed for proliferation and SC differentiation [41]. Within the first days, MPCS stimulated a provisional repair of the defect allowing the formation of a fibrin clot that is infiltrated by blood cells and fibroblasts. This created an important granulated tissue that joins the proximal and distal stump margins together, and acts as a matrix through which SCs and axons can migrate, also ensuring an micro-environment enriched by growth factors that stimulate axon/glia interactions and the wound angiogenic response [27]. De novo collagen synthesis was induced, as documented by gene expression analysis, by the increased expression of Tgf- $\beta 1$, which is fundamental for new collagen-rich matrix remodeling [42], together with a prompt restoration of a functional vasculature supported by the increased expression of Vegf mRNA [27].

After nerve injury, SCs undergo significant changes in cytoskeletal organization and molecular expression directed by their interaction with axons and adhesion to basal lamina [43]. Denervated SCs express Gfap marker and decrease the synthesis of myelin-related proteins [44]. In agreement, Gfap mRNA was upregulated in early phases, confirming the presence of many SCs in MPCS. Of note, the Gfap expression was down-regulated in MPCS group at 40 days, suggesting that SCs have already differentiated into a myelinating phenotype. Recent work has highlighted the role of EphB signaling between SC and fibroblast in nerve regeneration [28]. Interestingly, we observed that ephrin-B ligands are upregulated in MPCS, suggesting a coordinated action of SCs and fibroblasts in induced regeneration.

Axon/SC interaction is fundamental for successful regeneration and remyelination [45]. In MPCS the gap length had been crossed early by unmyelinated axons, elongated from the proximal stump, and was already in contact with SC after 15 days. Consistently, many key molecules required for correct SC/axon interactions,
A

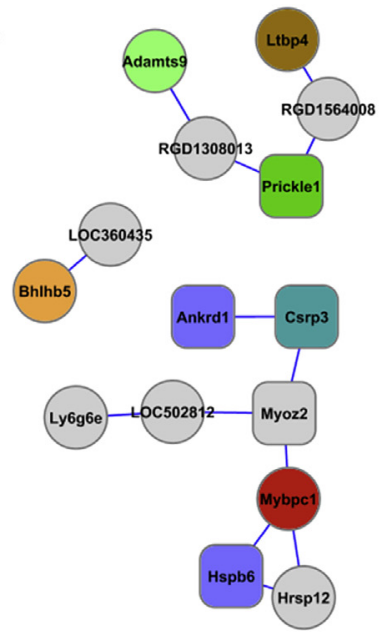

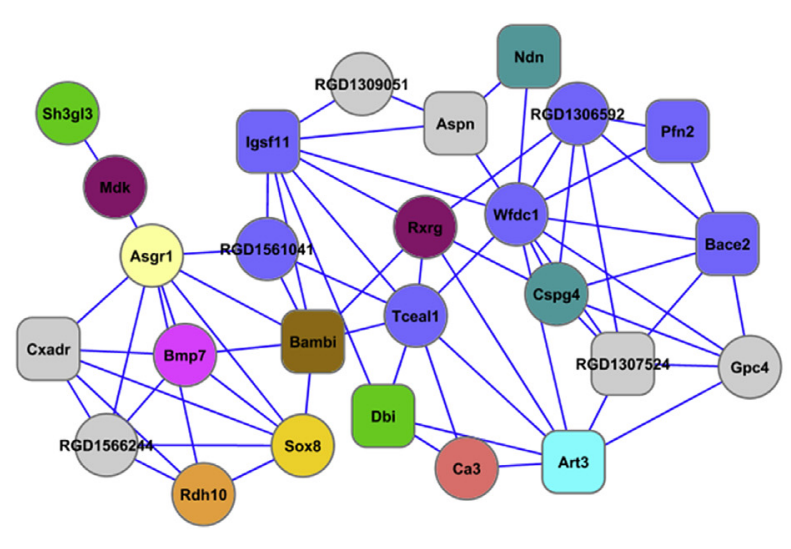

B

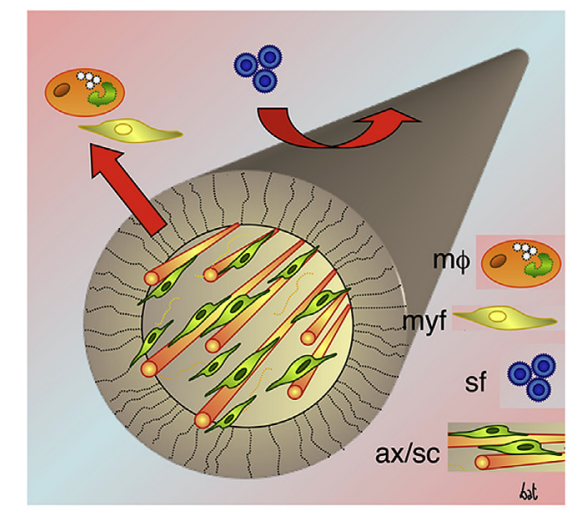

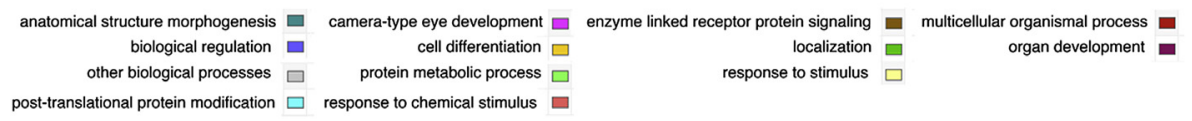

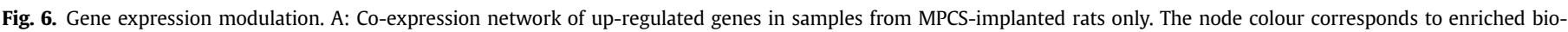

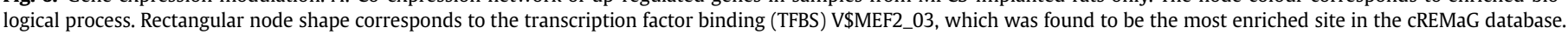

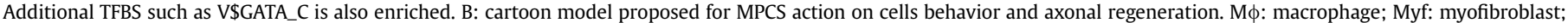

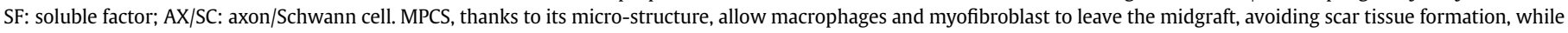
soluble factors as growth factors may enter into the scaffold and promote axonal regeneration and ultimately leading to normal myelinated fibers. 
including laminin receptors, integrins, dystroglycan and basal lamina components, were up-regulated in MPCS allowing successful nerve regeneration and remyelination.

In other experimental procedures as well as in our control group, axons show a delay in the process of myelination, even at 120 days. In MPCS instead, at the latest analyzed time point we observed a normal PNS development, consisting of normal axon diameters and myelin thickness. Although a strong downregulation of $\mathrm{Mpz}, \mathrm{Mbp}$ and Pmp22, the most representative myelin proteins in PNS, was expected at the early time points, the up-regulation of the myelin specific-gene was present only in crush injury and MPCS groups. This suggests that a molecular environment favorable for SCs differentiation was recreated in our scaffold. At 40 days, myelin genes decreased to normal levels, indicating that the process of myelination was completed. In postnatal development, axons control the SC myelination, optimizing myelin thickness to the axonal NRG1 type-III levels $[33,34]$. NRG1 binds to the glial ERBB2/B3 receptors, activating signal pathways, ultimately leading to the activation of the pro-myelinating transcription factor Krox20 [45]. SCs redifferentiate and the remyelinate newly formed axons in NRG1-dependent manner [46] while ERB2 is fundamental to guarantee SC survival and proliferation after injury [47]. Of note, ErbB2/B3 expression was up-regulated in MPCS group, further supporting the idea that MPCS enhances the regeneration processes. Our results suggest that the MPCS micro-structure stimulates cell behavior, from cell motility to activation and modulation of extracellular and intracellular signaling pathways, ultimately leading to changes in GE of molecules involved in SCs behavior and ECM formation. A direct comparison of differentially regulated genes between MPCS and CCNG showed an up-regulation in genes with a determinant role in SC development. We found that Sox10, a major player in the regulation of SC development [45], was upregulated in MPCS implanted animals.

Although a number of conduits permit the axonal elongation, there is no clear experimental evidence supporting the formation of functional and physiological endoneurium or perineurium. A speculative mechanism by which such a porous structure of MPCS may facilitate or promote regeneration is provided by the scaffold's capability to host and retain myofibroblasts. Indeed it has been suggested in the literature that the presence of an appropriate porosity allows contractile myofibroblast to attach to and migrate through the scaffold, thus limiting the contraction and the synthesis of scar tissue at the wound site [4,7]. Our data showed that inflammatory cells and myofibroblasts inside the MPCS, were likely to migrate from the cell-permeable inner tube lumen towards the cell-impermeable outer edge of the tube wall, also due to the radial orientation of porosity. MPCS micro-structure thus seems to prevent the formation of the contractile capsule of myofibroblasts and appears to be relevant in restoring a physiological perineurial nerve barrier and a normal endoneurium. In this situation, Dhh plays a relevant role in determining structural and functional characteristics of PNS connective tissue [32]. In humans, defects in Dhh cause a minifascicular neuropathy, characterized by the presence of extensive minifascicles within the endoneurium [48]. MPCS, through the regulation of Dhh, allows the development of a normal endoneurium, without formation of extensive minifascicles as observed in controls.

Analysis of GE data also demonstrated that a set of genes is differentially regulated in MPCS conduits only. Among the differentially expressed genes, we found those involved with cell cycle regulation, differentiation and proliferation. In MPCS, SCs are conducted into a regeneration pathway by the presence of relevant morphogenetic signals, such as Bone Morphogenetic Proteins (Bmp) [49]. Of note, Bmp is related to the Tgfb and Hedgehog signaling pathway $[50,51]$, including Dhh. It is well known that
Tgfb-receptor signaling mediates SC death and proliferation during nerve development [52,53], and similarly, hedgehog protein is relevant for morphogenesis of CNS and PNS [32,54]. Wnt signaling plays a major role in normal embryonic development, including the nervous system [55]. Interestingly MPCS up-regulated genes related to Wnt pathway like Dact1 (Dapper antagonist of catenin; Dapper/Frodo), Tmem88 (transmembrane protein 88) and Gpr177 (Wntless/Evi)). Dact1 was shown to be involved in the regulation of Wnt/b-catenin signaling [56] such as Tmem88 [57]. Similarly, GPR177 is a Wntless protein that is essential for proper sorting and secretion of WNT during development $[58,59]$. Wnt/b-Catenin signaling was shown to operate as a key driver of myelin gene expression and myelination both in PNS and CNS [40] and, of note, the canonical WNT signaling has been recently described as a key component of axonal sorting and lineage progression in Schwann cell development, operating in a very limited time window during mice embryonic development [60].

In conclusion, our findings provide ultrastructural and molecular evidences that the optimal micro-structure and degradation rate of collagen scaffold plays a key role in turning the inner microenvironment of injured nerves hospitable. We can infer that the porous structure and the collagen resorption rate of MPCS might recreate a favorable and well balanced cellular and molecular environment that allows PNS regeneration. Within these optimal conditions, the transected nerve recapitulates its innate molecular programs of differentiation in a coordinated fashion, which then ultimately leads to progressive substitution of non-neuronal with close to normal neuronal tissues.

\section{Acknowledgments}

This work was supported by "Fondo per gli Investimenti della Ricerca di Base" (FIRB-TissueNet grant) and PON-RINOVATIS (02_00563_3448479) to A.Q. and A.S.

\section{Appendix A. Supplementary data}

Supplementary data related to this article can be found at http:// dx.doi.org/10.1016/j.biomaterials.2014.01.069.

\section{References}

[1] Schröder JM. Altered ratio between axon diameter and myelin sheath thickness in regenerated nerve fibers. Brain Res 1972;45:49-65.

[2] Place ES, Evans ND, Stevens MM. Complexity in biomaterials for tissue engineering. Nat Mater 2009;28:457-70.

[3] Evans PJ. The peripheral nerve allograft: a decade of advancement. Atlas Hand Clin 2005; 10:187-97.

[4] Yannas IV. Emerging rules for inducing organ regeneration. Biomaterials 2013;34:321-30.

[5] Deumens R, Bozkurt A, Meek MF, Marcus MA, Joosten EA, Weis J, et al Repairing injured peripheral nerves: bridging the gap. Prog Neurobiol 2010;92:245-76.

[6] Chamberlain LJ, Yannas IV, Hsu HP, Spector M. Connective tissue response to tubular implants for peripheral nerve regeneration: the role of myofibroblasts. J Comp Neurol 2000;417:415-30.

[7] Soller EC, Tzeranis DS, Miu K, So PTC, Yannas IV. Common features of optimal collagen scaffolds that disrupt wound contraction and enhance regeneration both in peripheral nerves and in skin. Biomaterials 2012:33:4738-91.

[8] Yannas IV. Tissue and organ regeneration in adults. New York: Springer; 2001.

[9] Moore MJ, Friedman JA, Lewellyn EB, Mantila SM, Krych AJ, Ameenuddin S et al. Multiple-channel scaffolds to promote spinal cord axon regeneration. Biomaterials 2006:27:419-29.

[10] Stokols S, Tuszynski MH. Freeze-dried agarose scaffolds with uniaxial channels stimulate and guide linear axonal growth following spinal cord injury. Biomaterials 2006;27:443-51.

[11] Harley BA, Spilker MH, Wu JW, Asano K, Hsu H-P, Spector M, et al. Optima degradation rate for collagen chambers used for regeneration of periphera nerves over long gaps. Cells Tissues Organs 2004:176(1-3):153-65.

[12] Oh SH, Lee JH. Fabrication and characterization of hydrophilized porous PLGA nerve guide conduits by a modified immersion precipitation method. J Biomed Mater Res 2007;80:530-8. 
[13] Jeffries EM, Wang Y. Biomimetic micropatterned multi-channel nerve guides by templated electrospinning. Biotechnol Bioeng 2012;109:1571-82.

[14] Meek MF, Den Dunnen WF. Porosity of the wall of a Neurolac nerve conduit hampers nerve regeneration. Microsurgery 2009;29:473-8.

[15] Harley BA, Hastings AZ, Yannas IV, Sannino A. Fabricating tubular scaffolds with a radial pore size gradient by a spinning technique. Biomaterials 2006;27:866-74

[16] Quattrini A, Previtali S, Feltri ML, Canal N, Nemni R, Wrabetz L. Beta 4 integrin and other Schwann cell markers in axonal neuropathy. Glia 1996:17:294-306.

[17] Stoll G, Muller HW. Nerve injury, axonal de generation and neural regeneration: basic insights. Brain Pathol 1999;9:313-25.

[18] Triolo D, Dina G, Lorenzetti I, Malaguti M, Morana P, Del Carro U, et al. Loss of glial fibrillary acidic protein (GFAP) impairs Schwann cell proliferation and delays nerve regeneration after damage. J Cell Sci 2006;119:3981-93.

[19] La Marca R, Cerri F, Horiuchi K, Bachi A, Feltri ML, Wrabetz L, et al. TACE (ADAM17) inhibits Schwann cell myelination. Nat Neurosci 2011:14:857-65.

[20] Du P, Kibbe WA, Lin SM. Lumi: a pipeline for processing Illumina microarray. Bioinformatics 2008;24:1547-8.

[21] Wettenhall JM, Smyth GK. LimmaGUI: a graphical user interface for linear modeling of microarray data. Bioinformatics 2004:20:3705-6.

[22] Dennis Jr G, Sherman BT, Hosack DA, Yang J, Gao W, Lane HC, et al. DAVID: database for annotation, visualization, and integrated discovery. Genome biol 2003;4:P3.

[23] Huang da W, Sherman BT, Lempicki RA. Systematic and integrative analysis of large gene lists using DAVID bioinformatics resources. Nat Protoc 2009;4:44-57.

[24] Ashburner M, Ball CA, Blake JA, Botstein D, Butler H, Cherry JM, et al. Gene ontology: tool for the unification of biology. The Gene Ontology Consortium. Nat Genet 2000;25:25-9.

[25] Prifti E, Zucker JD, Clement K, Henegar C. FunNet: an integrative tool for exploring transcriptional interactions. Bioinformatics 2008;24:2636-8.

[26] Piechota M, Korostynski M, Solecki W, Gieryk A, Slezak M, Bilecki W, et al. The dissection of transcriptional modules regulated by various drugs of abuse in the mouse striatum. Genome Biol 2010;11:R48.

[27] Sondell M, Lundborg G, Kanje M. Vascular endothelial growth factor has neurotrophic activity and stimulates axonal outgrowth, enhancing cell survival and Schwann cell proliferation in the peripheral nervous system. J Neurosci 1999;19:5731-40.

[28] Parrinello S, Napoli I, Ribeiro S, Wingfield Digby P, Fedorova M, Parkinson DB, et al. EphB signaling directs peripheral nerve regeneration through Sox2dependent Schwann cell sorting. Cell 2010;143:145-55.

[29] Doyu M, Sobue G, Ken E, Kimata K, Shinomura T, Mitsuma T, et al. Laminin A $\mathrm{B} 1$, and $\mathrm{B} 2$ chain gene expression in transected and regenerating nerves: regulation by axonal signals. J Neurochem 1993;60:543-51.

[30] Nodari A. Beta1 integrin activates Rac1 in Schwann cells to generate radia lamellae during axonal sorting and myelination. J Cell Biol 2007;177:1063-75.

[31] Feltri ML, Scherer SS, Nemni R, Kamholz J, Vogelbacker H, Scott MO, et al. Beta 4 integrin expression in myelinating Schwann cells is polarized, developmentally regulated and axonally dependent. Development 1994;120:1287-301.

[32] Parmantier E, Lynn B, Lowson D, Turmaine M, Namini SS, Chakrabarti L, et al Schwann cell-derived Desert hedgehog controls the development of peripheral nerve sheaths. Neuron 1999;23:713-24.

[33] Michailov GV, Sereda MW, Brinkmann BG, Fischer TM, Haug B, Birchmeir C, et al. Axonal neuregulin-1 regulates myelin sheath thickness. Science 2004;304:700-3.

[34] Taveggia C, Zanazzi G, Petrylak A, Yano H, Rosenbluth J, Einheber S, et al Neuregulin-1 type III determines the ensheathment fate of axons. Neuron 2005; 47:681-94.

[35] Greenfield S, Brostoff A, Eylar EH, Morell P. Protein composition of myelin of the peripheral nervous system. J Neurochem 1973;20:1207-16.

[36] LeBlanc AC, Poduslo JF. Axonal modulation of myelin gene expression in the peripheral nerve. J Neurosci Res 1990;26:317-26.

[37] Nishino J, Yamashita K, Hashiguchi H, Fujii H, Shimazaki T, Hamada H Meteorin: a secreted protein that regulates glial cell differentiation and promotes axonal extension. EMBO J 2004;23:1998-2008.
[38] Eshed Y. Gliomedin mediates Schwann cell-axon interaction and the molecular assembly of the Nodes of Ranvier. Neuron 2005;47:215-29.

[39] Zhelyaznik N, Mey J. Regulation of retinoic acid receptors alpha, beta and retinoid X receptor alpha after sciatic nerve injury. Neuroscience 2006;141: $1761-74$.

[40] Tawk M, Makoukji J, Belle M, Fonte C, Trousson A, Hawkins T, et al. Wnt/BCatenin signaling is an essential and direct driver of myelin gene expression and myelinogenesis. J Neurosci 2011;31:3729-42.

[41] Bosse F, Hasenpusch-Theil K, Kury P, Muller HW. Gene expression profiling reveals that peripheral nerve regeneration is a consequence of both novel injury-dependent and reactivated developmental processes. J Neurochem 2006;96:441-1457.

[42] Einheber S, Hannocks MJ, Metz CN, Rifkin DB, Salzer JL. Transforming growth factor-beta 1 regulates axon/Schwann cell interactions. J Cell Biol 1995;129: 443-58.

[43] Podratz JL, Rodriguez E, Windebank AJ. Role of the extracellular matrix in myelination of peripheral nerve. Glia 2001;35:35-40.

[44] Scherer SS. The biology and pathobiology of Schwann cells. Curr Opin Neurol $1997 ; 10: 386-97$

[45] Svaren J, Meijer D. The molecular machinery of myelin gene transcription in Schwann cells. Glia 2008;56:1541-51.

[46] Fricker FR, Lago N, Balarajah S, Tsantoulas C, Tanna S, Zhu N, et al. Axonally derived neuregulin-1 is required for remyelination and regeneration after nerve injury in adulthood. J Neurosci 2011;31:3225-33.

[47] Atanasoski S, Scherer SS, Sirkowski E, Leone D, Garratt AN, Birchmeier C, et al. ErbB2 signaling in Schwann cells is mostly dispensable for maintenance of myelinated peripheral nerves and proliferation of adult Schwann cells after injury. J Neurosci 2006;26:2124-31.

[48] Umehara F, Tate G, Itoh K, Osame M. Minifascicular neuropathy: a new concept of the human disease caused by desert hedgehog gene mutation. Cell Mol Biol 2002;48:187-9.

[49] Chen D, Zhao M, Mundy GR. Bone morphogenetic proteins. Growth Factors 2004;22:233-41.

[50] Charon F, Tessier-Lavigne M. Novel brain wiring functions for classical morphogens: a role as graded positional cues in axon guidance. Development 2005; $132: 2251-62$.

[51] Feng XH, Derynck R. Specificity and versatility in tgf-beta signaling through Smads. Annu Rev Cell Dev Biol 2005;21:659-93.

[52] Parkinson DB, Dong Z, Bunting H, Whitfield J, Meier C, Marie H, et al. Transforming growth factor beta (TGFbeta ) mediates Schwann cell death in vitro and in vivo: examination of c-Jun activation, interactions with survival signals, and the relationship of TGF-beta mediated death to Schwann cell differentiation. J Neurosci 2001;21:8572-85.

[53] D’Antonio M, Droggiti A, Feltri ML, Roes J, Wrabetz L, Mirsky R, et al. TGFbeta type II receptor signaling controls Schwann cell death and proliferation in developing nerves. J Neurosci 2006;26:8417-27.

[54] Goodrich L, Scott M. Hedgehog and patched in neural development and disease. Neuron 1998;21:1243-57.

[55] Ciani L, Salinas PC. WNTs in the vertebrate nervous system: from pattering to neuronal connectivity. Nat Rev Neurosci 2005;6:351-62.

[56] Cheyette BN, Waxman JS, Miller JR, Takemaru K, Sheldahl LC, Khlebtsova N, et al. Dapper, a Dishevelled-associated antagonist of beta-catenin and JNK signaling, is required for notochord formation. Dev Cell 2002;2:449-61.

[57] Lee HJ, Finkelstein D, Li X, Wu D, Shi DL, Zheng JJ. Identification of transmembrane protein 88 (TMEM88) as a dishevelled-binding protein. J Biol Chem 2010;285:41549-56.

[58] Bänziger C, Soldini D, Schütt C, Zipperlen P, Hausmann G, Basler K. Wntless, a conserved membrane protein dedicated to the secretion of Wht proteins from signaling cells. Cell 2006;125:509-22.

[59] Bartscherer K, Pelte N, Ingelfinger D, Boutros M. Secretion of Wnt ligands requires Evi, a conserved transmembrane protein. cell 2006;125:523-33.

[60] Grigoryan T, Stein S, Qi J, Wende H, Garratt AN, Nave KA, et al. Wnt/Rspondin/ $\beta$-catenin signals control axonal sorting and lineage progression in Schwann cell development. Proc Natl Acad Sci USA 2013;110(45):18174-9. 